\title{
Crystal structure of 4'-hydroxy-5,6-benzoflavone, $\mathrm{C}_{19} \mathrm{H}_{12} \mathrm{O}_{3}$
}

\author{
M. Shoja ${ }^{* . l}$, A. Krikava ${ }^{\mathrm{I}}$ and R. Kabbani ${ }^{\text {II }}$ \\ I Fordham University, Department of Chernistry, Fordham Road, Bronx, NY 10458, USA \\ " Pace University, Department of Chemistry and Physical Sciences, One Pace Plaza, New York, NY 10038, USA
}

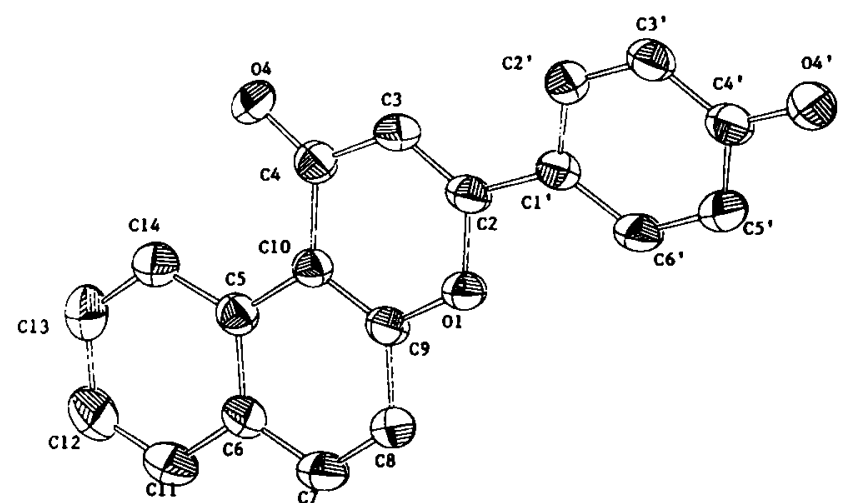

\section{Abstract}

$\mathrm{C}_{19} \mathrm{H}_{12} \mathrm{O}_{3}$, monoclinic, $P 12_{1} / c 1$ (No. 14), $a=8.157(4) \AA$,

$b=15.091(2) \AA, c=11.035(2) \AA, \beta=92.37(3)^{\circ}, V=1357.2 \AA^{3}$, $Z=4, R_{\mathrm{gt}}(F)=0.060, w R_{\mathrm{all}}(F)=0.088, T=293 \mathrm{~K}$.

\section{Source of material}

Crystals of the title compound (Indofine Chemical Company) were grown from ethyl acetate solution, $\mathrm{mp} 576 \mathrm{~K}-578 \mathrm{~K}$.

\section{Discussion}

$\mathrm{X}$-ray analysis has shown that 5,6-benzoflavone is planar with a dihedral angle of $1.07^{\circ}$. The planarity of the molecule was considered to be due to the stabilization of the negative charge on the carbonyl $\mathrm{O}$ atom [1]. Potential energy calculations for the same molecule predicted a non-planar conformation. We have reported in the past the same type of disagreement between molecular quantum mechanical calculations and $X$-ray results [2]. The $X$-ray single crystal structure of 4'-hydroxy- $\beta$-naphthoflavone was undertaken to show that the conformation of these types of molecules are affected primarily by the crystal packing [3]. The title compound is in a non-planar conformation. The dihedral angle is $18.0(2)^{\circ}$, and $d\left(\mathrm{C} 2-\mathrm{Cl}^{\prime}\right)=1.440(8) \AA$.
Table 1. Data collection and handling.

$\begin{array}{ll}\text { Crystal: } & \text { colorless needle, size } 0.2 \times 0.2 \times 0.3 \mathrm{~mm} \\ \text { Wavelength: } & \text { Mo } K_{\alpha} \text { radiation }(0.71073 \AA) \\ \mu: & 1.0 \mathrm{~cm}^{-1} \\ \text { Diffractometer, scan mode: } & \text { CAD4, } \omega / 2 \theta \\ 2 \theta_{\max }: & 49.94^{\circ} \\ N(h k l)_{\text {measured, }} N(h k l)_{\text {unique: }}: & 2725,2376 \\ \text { Criterion for } F_{\text {obs, }} N(h k l)_{\text {g: }}: & F_{\text {obs }}>3 \sigma\left(F_{\text {obs }}\right), 1351 \\ N(\text { param })_{\text {refined: }} & 247 \\ \text { Program: } & \text { XTAL [4] }\end{array}$

Table 2. Atomic coordinates and displacement parameters (in $\AA^{\mathbf{2}}$ ).

\begin{tabular}{llllll}
\hline Atom & Site & $x$ & $y$ & $z$ & $U_{\text {iso }}$ \\
\hline H(6') & $4 e$ & $0.120(6)$ & $0.222(3)$ & $0.914(5)$ & $0.03(1)$ \\
H(3') & $4 e$ & $-0.201(7)$ & $0.220(4)$ & $1.254(5)$ & $0.05(2)$ \\
H(2') & $4 e$ & $-0.073(7)$ & $0.345(4)$ & $1.202(5)$ & $0.07(2)$ \\
H(13) & $4 e$ & $0.565(7)$ & $0.781(4)$ & $0.905(5)$ & $0.05(2)$ \\
H(8) & $4 e$ & $0.222(7)$ & $0.399(4)$ & $0.693(5)$ & $0.05(2)$ \\
H(5') & $4 e$ & $-0.011(7)$ & $0.095(4)$ & $0.975(5)$ & $0.06(2)$ \\
H(12) & $4 e$ & $0.626(8)$ & $0.766(4)$ & $0.715(5)$ & $0.07(2)$ \\
H(14) & $4 e$ & $0.436(7)$ & $0.674(4)$ & $1.004(5)$ & $0.05(2)$ \\
H(3) & $4 e$ & $0.152(8)$ & $0.441(4)$ & $1.178(6)$ & $0.07(2)$ \\
H(7) & $4 e$ & $0.370(7)$ & $0.506(4)$ & $0.604(6)$ & $0.06(2)$ \\
H(11) & $4 e$ & $0.513(7)$ & $0.644(4)$ & $0.614(5)$ & $0.05(2)$ \\
H(4') & $4 e$ & $-0.17(1)$ & $0.013(3)$ & $1.132(7)$ & $0.20(3)$ \\
& & & & & \\
\hline
\end{tabular}

Table 3. Atomic coordinates and displacement parameters (in $\AA^{2}$ ).

\begin{tabular}{|c|c|c|c|c|c|c|c|c|c|c|}
\hline Atom & Site & $x$ & $y$ & $z$ & $U_{11}$ & $U_{22}$ & $U_{33}$ & $U_{12}$ & $U_{13}$ & $U_{23}$ \\
\hline$C(10)$ & $4 e$ & $0.2978(6)$ & $0.5217(3)$ & $0.9303(4)$ & $0.037(3)$ & $0.033(3)$ & $0.029(3)$ & $0.004(2)$ & $0.005(2)$ & $0.001(2)$ \\
\hline$O(1)$ & $4 e$ & $0.1580(5)$ & $0.3814(2)$ & $0.9053(3)$ & $0.053(2)$ & $0.041(2)$ & $0.029(2)$ & $-0.008(2)$ & $0.005(2)$ & $-0.001(2)$ \\
\hline$C(2)$ & $4 e$ & $0.1217(7)$ & $0.3784(4)$ & $1.0250(4)$ & $0.042(3)$ & $0.043(3)$ & $0.028(3)$ & $0.005(3)$ & $0.005(2)$ & $0.005(2)$ \\
\hline$C(9)$ & $4 e$ & $0.2403(7)$ & $0.4518(3)$ & $0.8603(4)$ & $0.042(3)$ & $0.040(3)$ & $0.029(3)$ & $0.002(3)$ & $0.002(2)$ & $0.004(2)$ \\
\hline$C(5)$ & $4 e$ & $0.3878(7)$ & $0.5910(3)$ & $0.8719(5)$ & $0.039(3)$ & $0.037(3)$ & $0.034(3)$ & $0.005(3)$ & $0.004(2)$ & $0.003(2)$ \\
\hline$C(4)$ & $4 e$ & $0.2630(7)$ & $0.5202(4)$ & $1.0609(5)$ & $0.041(3)$ & $0.038(3)$ & $0.034(3)$ & $0.008(3)$ & $0.009(2)$ & $0.001(3)$ \\
\hline$C(3)$ & $4 e$ & $0.1723(7)$ & $0.4454(4)$ & $1.0991(5)$ & $0.049(4)$ & $0.045(3)$ & $0.026(3)$ & $0.001(3)$ & $0.009(3)$ & $-0.000(3)$ \\
\hline
\end{tabular}

* Correspondence author (e-mail: shoja@ fordham.edu) 
Table 3. Continued.

\begin{tabular}{llrllllrrrr}
\hline Atom & Site & \multicolumn{1}{c}{$\boldsymbol{x}$} & $y$ & $z$ & $U_{11}$ & $U_{22}$ & $U_{33}$ & $U_{12}$ \\
\hline $\mathrm{O}(4)$ & $4 e$ & $0.3120(5)$ & $0.5784(3)$ & $1.1331(3)$ & $0.071(3)$ & $0.039(2)$ & $0.036(2)$ & $-0.006(2)$ & $0.011(2)$ & $-0.010(2)$ \\
$\mathrm{C}(8)$ & $4 e$ & $0.2626(8)$ & $0.4456(4)$ & $0.7350(5)$ & $0.060(4)$ & $0.042(4)$ & $0.032(3)$ & $-0.006(3)$ & $0.001(3)$ & $-0.002(3)$ \\
$\mathrm{C}(11)$ & $4 e$ & $0.4968(9)$ & $0.6509(5)$ & $0.6865(6)$ & $0.082(5)$ & $0.062(5)$ & $0.037(4)$ & $-0.008(4)$ & $0.014(3)$ & $0.008(4)$ \\
$\mathrm{C}\left(1^{\prime}\right)$ & $4 e$ & $0.0402(7)$ & $0.2974(3)$ & $1.0560(5)$ & $0.037(3)$ & $0.039(3)$ & $0.030(3)$ & $-0.001(3)$ & $-0.001(2)$ & $0.003(2)$ \\
$\mathrm{C}(14)$ & $4 e$ & $0.4514(8)$ & $0.6667(4)$ & $0.9316(6)$ & $0.047(4)$ & $0.045(4)$ & $0.038(3)$ & $-0.001(3)$ & $0.006(3)$ & $-0.000(3)$ \\
$\mathrm{C}(6)$ & $4 e$ & $0.4106(7)$ & $0.5833(4)$ & $0.7455(5)$ & $0.050(4)$ & $0.041(3)$ & $0.035(3)$ & $-0.002(3)$ & $0.008(3)$ & $0.005(3)$ \\
$\mathrm{C}\left(3^{\prime}\right)$ & $4 e$ & $-0.1257(8)$ & $0.2154(4)$ & $1.1930(5)$ & $0.049(4)$ & $0.047(4)$ & $0.034(3)$ & $0.003(3)$ & $0.006(3)$ & $0.007(3)$ \\
$\mathrm{C}(13)$ & $4 e$ & $0.5326(8)$ & $0.7301(4)$ & $0.8705(6)$ & $0.062(5)$ & $0.037(4)$ & $0.060(4)$ & $-0.007(3)$ & $0.003(3)$ & $-0.001(3)$ \\
$\mathrm{C}\left(2^{\prime}\right)$ & $4 e$ & $-0.0456(7)$ & $0.2908(4)$ & $1.1638(5)$ & $0.046(4)$ & $0.038(3)$ & $0.035(3)$ & $0.004(3)$ & $0.005(3)$ & $-0.000(3)$ \\
$\mathrm{C}(7)$ & $4 e$ & $0.3447(8)$ & $0.5104(4)$ & $0.6802(5)$ & $0.066(4)$ & $0.056(4)$ & $0.029(3)$ & $-0.006(3)$ & $0.006(3)$ & $0.002(3)$ \\
$\mathrm{O}\left(4^{\prime}\right)$ & $4 e$ & $-0.2143(7)$ & $0.0686(3)$ & $1.1404(4)$ & $0.098(4)$ & $0.048(3)$ & $0.045(2)$ & $-0.020(3)$ & $0.015(2)$ & $0.002(2)$ \\
$\mathrm{C}\left(4^{\prime}\right)$ & $4 e$ & $-0.1311(7)$ & $0.1431(4)$ & $1.1160(5)$ & $0.056(4)$ & $0.047(4)$ & $0.034(3)$ & $-0.008(3)$ & $-0.003(3)$ & $0.005(3)$ \\
$\mathrm{C}(12)$ & $4 e$ & $0.5570(9)$ & $0.7225(5)$ & $0.7466(6)$ & $0.069(5)$ & $0.052(4)$ & $0.055(4)$ & $-0.009(4)$ & $0.013(4)$ & $0.013(3)$ \\
$\mathrm{C}\left(6^{\prime}\right)$ & $4 e$ & $0.0373(8)$ & $0.2239(4)$ & $0.9807(5)$ & $0.061(4)$ & $0.049(4)$ & $0.028(3)$ & $-0.009(3)$ & $0.009(3)$ & $0.003(3)$ \\
$\mathrm{C}\left(5^{\prime}\right)$ & $4 e$ & $-0.0432(9)$ & $0.1477(4)$ & $1.0096(5)$ & $0.073(5)$ & $0.047(4)$ & $0.034(3)$ & $-0.010(4)$ & $0.008(3)$ & $-0.004(3)$ \\
& & & & & & & & \\
\hline
\end{tabular}

\section{References}

1. Rossi, M.; Cantrell, J. S.; Farber, A. J.; Dyott, T.; Carrell, H. L.; Glusker, J. P.: Molecular structures of 5,6- and 7,8-benzoflavones, inhibitors of aryl hydrocarbon hydroxylase. Cancer Research 40 (1980) 2774-2784.

2. Shoja, M.; Bisso, S.; Kabbani, R.; Athanasopoulos, D.: Crystal stucture of 6-hydroxy-2'-methoxyflavone. Z. Kristallogr. NCS 214 (1999) 233-234.

3. Shoja, M.: Crystal structure of 3-hydroxy-6-methoxyflavone. $Z$. Kristallogr. NCS 213 (1998) 731-732.

4. Hall, S. R.; King, G. S. D.; Stewart, J. M. (Eds.): Xtal3.4 User's Manual. University of Western Australia: Lamb, Perth, Australia 1995. 